\title{
Competing Cosmology Models. Can Entropy Production Help Falsify Cyclic Models of Cosmology, or Variants along the Lines Discussed by Roger Penrose at the ICG Conference in Penn State, 2007?
}

\section{Andrew Walcott Beckwith}

Physics Department, College of Physics, Chongqing University Huxi Campus, Chongqing, China Email: Rwill9955b@gmail.com, abeckwith@uh.edu

How to cite this paper: Beckwith, A.W. (2016) Competing Cosmology Models. Can Entropy Production Help Falsify Cyclic Models of Cosmology, or Variants along the Lines Discussed by Roger Penrose at the ICG Conference in Penn State, 2007? Journal of High Energy Physics, Gravitation and Cosmology, 2, 486-505.

http://dx.doi.org/10.4236/jhepgc.2016.24043

Received: June 30, 2016

Accepted: August 21, 2016

Published: August 24, 2016

Copyright $\odot 2016$ by author and Scientific Research Publishing Inc. This work is licensed under the Creative Commons Attribution International License (CC BY 4.0).

http://creativecommons.org/licenses/by/4.0/

\section{Abstract}

In the inaugural ICG meeting, on August 11, 2007 at Penn State, Roger Penrose [1] gave a presentation about an alternative to cyclic cosmological models, which needs experimental tests for falsifiability. As discussed by Beckwith, in EJTP [2], Penrose brought up how wave equation, as simplified in flat space could lead to a rising vacuum nucleation field which would engender the pop up behavior as sought in most emergent field models of gravity. The scalar field pop up with certain qualifications is not so startling in itself. Now for the radical extension Penrose brought to bear. Penrose asserted in his ICG lecture that there was a good chance that there was no collapse in future events, but that matter would be eventually sucked up by "millions" of black holes, creating a clean-up of most interstellar matter. The issue to be brought up is how to come up with a mapping for re-combination of the black hole collected material, for a big bang, a topic which was not solved by Penrose.

\section{Keywords}

Entropy Production, Cosmology, Variants

\section{Introduction}

Penrose asserted that the "millions of black holes" would eventually undergo Hawking's evaporation [1], i.e. that in some fashion that there would be a release of the matterenergy. For those who wish to look it up, Hawking's evaporation of black holes, in- 
volves subtle quantum arguments and tries to reconcile black hole physics with known thermodynamics, for example the $2^{\text {nd }}$ law of Black hole dynamics. Traschen [3] states the basic assumptions involved, while Hawkings [4] stated evaporation as to ways which may tie in with typical entropy/area calculations as given by Bernstein and other writers. The easiest conceptual starting point is to use the equivalence between number of operations which Lloyd [5] used in his model, and total units of entropy as the author referenced from Carroll [6], and other theorists. The key equation Seth Lloyd [5] wrote is as follows, assuming a low entropy value in the beginning

$$
\left|S_{\text {Total }}\right| \sim\left|k_{B} \cdot \ln 2\right| \cdot[\text { \# operations }]^{3 / 4} \sim 10^{5}-10^{6} .
$$

Seth Lloyd [5] is making a direct reference to a linkage between the number of operations a quantum computer model of how the Universe evolves is responsible for, in the onset of a big bang picture, and entropy. Needless to state though, Equation (1) above, and the issue of that if or not there is a well defined threshold bulk electric and magnetic charge contribution to energy. If there is, indeed an evaporation effect of black hole physics, at what juncture does one have a collapse of a threshold effect for calculations about the minimum entropy based upon black hole models involving electric and magnetic charges?

Assuming then, that the relevant Black holes evaporate, Penrose [1] next presented the question of an undetermined mapping of the evaporated Hawking radiation back to the nexus point for a new big bang. The author, Beckwith, asked Penrose repeatedly at the ICG about the nature of the mapping of released Hawking radiation back to a new big bang. Penrose threw the question back to Beckwith, as Beckwith's research problem, not his. Assume, if one will that there are $\mathrm{N}$ number of universes undergoing Penrose style expansion and then black hole clean up of matter-energy as these $\mathrm{N}$ universes expand. Each universe contains roughly $10^{88}$ entropy units of computational information as embedded in say $10^{10}$ spiral galaxies. If each spiral galaxy has an entropy reading of about $10^{90}$ entropy "units", this leads to an overhang of about $10^{100}$ entropy units, as opposed to an observable $10^{88}$ entropy units for the universe as can be accessed by instrumentation. Which leads asking what is the significance of that entropy gap?

Secondly, and most important to this discussion, there is a strange attractor suck up of bits of information from each of the $\mathrm{N}$ expanding universes, and the Hawking radiation is, within a mega structure mapped back to the locus point of another set of $\mathrm{N}$ big bangs via typical phase space strange attractor dynamics. How to verify this wild supposition experimentally? See the conclusion of this article for Beckwith's guess as to what to try to do experimentally to indirectly infer the existence of this mega structure and of strange attractor collapse of Hawkings radiation back to $\mathrm{N}$ locus points for $\mathrm{N}$ number of big bangs.

\section{What Is Needed to Be Experimentally Falsified: Relic Graviton Production Involves HFGWs, Indicated by a Rapid Drop off of Graviton Creation after the Onset of the Big Bang}

We should first look at the key assumption of the $\mathrm{Ng}$ [7] [8] approach to entropy: the 
wavelength of the "particles" contributing to entropy are ultra-long, i.e., there is an order of magnitude difference between the cube of the wavelengths of the particles and of the containing volume of space, $\mathrm{V}$, which is analyzed to obtain the entropy figure $\mathrm{Ng}$ [7]

[8] uses to get his infinite quantum statistics. The same methodology of comparing the cube of wavelengths with the expected spacetime volume is used to get $\mathrm{Ng}$ 's [7] [8] infinite quantum statistics, assuming that relic graviton production involves HFGWs. Then one analyzes entropy production what $\mathrm{Ng}$ did with DM and wavelengths, and the volume of space $\mathrm{V}$,. But instead of DM, this involves gravitons, with an ultra-short wavelength, necessitating a small volume of space in the beginning of graviton production. So the same infinite quantum statistics procedure Ng used for DM can be used for gravitons, except that the gravitons are produced in the very beginning of the inflationary era. So the creation of gravitons is enhanced in the beginning of cosmological nucleation by the requirement of a one-to-one relationship between shortwave lengths of HFGW and a small space time volume for relic graviton creation. Then it's likely that the data sets observed in the Li-Baker detector could indicate a rapid drop off of graviton creation after the onset of the big bang. This should be investigated by falsifiable experimental procedures.

\section{Prediction: A Relatively Narrow Range of GW Frequencies for Relic Graviton Production}

Appendix $\mathrm{C}$ examines this assumption and compares it directly with another assumption made by Giovannini [9], which is reformulated to assert that if all frequency ranges for GW radiation were permissible, one would see a total value of entropy of nearly $10^{90}$. This is done while not assuming as we did HFGW conditions.

Therefore, Giovannini's (1993) prediction as written up in 2008 [9] is assumed to be indefensible, and that a relatively narrow range of GW frequencies for relic graviton production is what should be looked for via either the Li-Baker HFGW detector or by the Planck satellite mission.

\section{Implication: How an Inflation Could Arise and Fall from Thermal Inputs from a Prior Universe}

Here are some additional possible spinoffs of these sorts of ideas, if they are experimentally verified. Appendix D based upon Beckwith's work, [9] shows a to-the-point presentation of how an inflation could arise and fall from thermal inputs from a prior universe. These are notes adapted from a presentation by Dr. Penrose regarding his alternatives to typical cyclic-universe cosmologies [1]. We elaborate upon Penrose's startling conclusions, but his first part of his presentation is useful, since it fits very closely with the author's methodologies for thermal inputs from a prior universe.

\section{Are Irregularities in the CMBR Spectra Related to Entropy Production?}

If this can be verified experimentally, the biggest payoff would be to address an issue 
that the author discussed with Sarkar of Oxford [10]. Appendix A gives the basic idea: are the irregularities in the CMBR spectra, due to non-standard physics, which are an extension of the standard inflation model, used to justify entropy production? We think that there is merit to this idea and that it should be investigated. At the minimum, understanding entropy production would allow us to analyze if the structure formation methodology experimentally presented by Rtuu, et al. [11] ties in with models of entropy production, and if not, what about verifying the standard model for CMBR production, as G. Hingsaw [12] and others promote? Or what if Sarkar [11] is right? A summary of what A.W. Beckwith [13] thinks of these issues may be found in a presentation made at IDM 2008.

\section{Structure Formation from Entropy Generation}

Starting with what Beckwith used in 2008 [13], and also in Rencontres De Blois [14]

$$
\left|S_{\text {Total }}\right|_{\text {Initial-inf-condt }} \sim\left|k_{B} \cdot \ln 2\right| \cdot[\# \text { operations }]^{3 / 4} \approx N\left|-\log \frac{N}{10}+\log V_{4}^{3}+\log E^{3 / 2}\right| \sim 10^{5} \text {. }
$$

Aiding in the development of confirming/falsifying Equation (2) above are structure formation questions that we leave as open questions to be addressed by the CMBR/astrophysics community: This would be aligned with the question of how structure formation could arise as a result of entropy generation. Sarkar [11] and others, with their race track models of inflation, have done useful pioneering work in defining coupled fields undergoing symmetry breaking that are coupled to the inflation. The author, A.W. Beckwith, thinks that such suppositions need experimental verification, and that the boost of total entropy by the relic graviton value given in $\Delta S_{\text {graviton-production }} \propto 10^{5}$ in a Planck time interval could lead to additional insights into whether or not Sarkar [11] (2008) or Hingsaw [12] is right about the origins of irregularities in the CMBR spectra. Sarkar $\{11\}$ states that the irregularities means physics beyond the standard cosmological model assumed for WMAP, while Hingsaw [12] states that the irregularities are merely statistical anomalies.

\section{How Initially Huge Vacuum Energy and Its Rapid Collapse in Space-Time to a Much Smaller Cosmological Constant Value Aids in the Breakup and Reformulation of Entropy Production?}

The author, A.W. Beckwith, wishes to close with what will be future projects to address some of the above issues. As discussed with Tchrakian, [15] Bremen, August 29 ${ }^{\text {th }}, 2008$, the author wishes to determine if or not the dichotomy between an initially huge vacuum energy, as specified above in this manuscript, and its rapid collapse in space-time to a much smaller cosmological constant value, aids in the breakup and reformulation of entropy production. The author's supposition is that it is relevant to two areas. First, the author assume that there is a breakup of the initial instanton structure from a prior universe. Since the author also views gravitons as a kink-antikink structure, the supposition is that initially, from a prior to a present universe, there would be a similar phenomenon: initial lack of numerical density of gravitons just before a second-order 
phase transition, which is discussed in part in Appendix C. Secondly if, after a secondorder phase transition we see evidence of astrophysical data supporting the rebirth of both entropy and graviton production, we should take this hypothesis seriously. Should the cosmological constant/vacuum energy linkage be proved to be consistent with the breakup and then reformulation of graviton production in a phase transition, then the author, A.W. Beckwith, thinks that researchers could be on track for new experimentally falsifiable criteria, to be developed for CMBR physics.

\section{Finally, Relic Graviton Produced Entropy at the Onset of the Big Bang. Why Starting Entropy Would Be So Small While CMBR Entropy Would Be So Large}

As a closing remark, Beckwith wishes to suggest a solution to Penrose's implied question about entropy as raised in Edingborough, Scotland [16] conference proceedings. Penrose talks about the $2^{\text {nd }}$ law, and its implied requirements as to the small initial value of early universe entropy, and then states that gravitational entropy would not be so major, whereas CMBR matter contributed entropy would be much larger. Beckwith is convinced that relic graviton production at the onset of the big bang, i.e. before the contribution of entropy from matter itself would be necessary to boost entropy from its small $10^{5}$ value at the onset of the big bang, to a much higher level, and that entropy would be initially dramatically boosted by that process. i.e. the uniformity requirement Penrose talks about in structure would be actually as of up to the Electro weak transition, and far after the initial onset of inflation itself.

Sanchez, in [17] also elaborates upon the case for ultra-low entropy in [17]. The author does not completely agree with the diagnosis, but it is standard.

\section{A New Idea Extending Penrose's Suggestion of Cyclic Universes, Black Hole Evaporation, and the Embedding Structure Our Universe Is Contained within}

Beckwith strongly suspects that there are no fewer than $\mathrm{N}$ (a large number) of universes undergoing Penrose "infinite expansion" and all these are contained within a mega universe structure. Furthermore, that each of the $\mathrm{N}$ universes has black hole evaporation commencing, with the Hawking radiation from decaying black holes.

If each of the $N$ universes is definable by a partition function, we can call $\left\{\Xi_{i}\right\}_{i=N}^{i=1}$, then there exist an information minimum ensemble of mixed minimum information roughly correlated as about $10^{7}-10^{8}$ bits of information per each partition function in the set $\left.\left\{\Xi_{i}\right\}_{i=N}^{i=1}\right|_{\text {before }}$, so minimum information is conserved between a set of partition functions per each universe

$$
\left.\left.\left\{\Xi_{i}\right\}_{i=N}^{i=1}\right|_{\text {before }} \equiv\left\{\Xi_{i}\right\}_{i=N}^{i=1}\right|_{\text {after }} .
$$

However, that there is non uniqueness of information put into each partition function $\left\{\Xi_{i}\right\}_{i=N}^{i=1}$. Furthermore that within the mega structure, that Hawking radiation from the black holes is collated via a strange attractor collection in the mega universe 
structure to form a new big bang for each of the $\mathrm{N}$ universes as represented by $\left\{\Xi_{i}\right\}_{i=N}^{i=1}$. Verification of this mega structure compression and expansion of information with a non unique venue of information placed in each of the $\mathrm{N}$ universes would strongly favor Ergodic mixing treatments of initial values for each of the $\mathrm{N}$ universes expanding from a quasi singularity beginning. If this idea is in any way confirmable, it would lend credence as to the formation of the dark flow hypothesis, and of how an harmonic perturbative contributions to initial inflationary expansion may occur, within a partially random ergotic background. Beckwith claims that such a process would inherently favor the small $10^{7}$ bits of information per each partition function representing the "start" of expansion of a new universe. Hopefully, in doing so, one can explain, eventually, the problems with entropy modeling presented in Appendix $\mathrm{C}$ below. This has a similarity with a construction done by Beckwith [18], namely looking at the following expression of energy flux being re-formulated for each universe. i.e. start with the Alcubierre's formalism about energy flux, assuming that there is a solid angle for energy distribution $\Omega$ for the energy flux to travel through [18].

$$
\frac{\mathrm{d} E}{\mathrm{~d} t}=[\lim r \rightarrow \infty]\left[\frac{r^{2}}{16 \pi}\right] \oint\left|\int_{-\infty}^{t} \Psi_{4} \mathrm{~d} t^{\prime}\right|^{2} \cdot \mathrm{d} \Omega
$$

The expression $\Psi_{4}$ is a Weyl scalar which we will write in the form of

$$
\Psi_{4}=-\frac{1}{4} \cdot\left[\partial_{t}^{2} h^{+}-2 \partial_{t} \partial_{r} h^{+}+\partial_{r}^{2} h^{+}\right]+\frac{i}{4} \cdot\left[\partial_{t}^{2} h^{x}-2 \partial_{t} \partial_{r} h^{x}+\partial_{r}^{2} h^{x}\right] .
$$

Our assumptions are simple, that if the energy flux expression is to be evaluated properly, before the electro weak phase transition, that time dependence of both $h^{+}$ and $h^{x}$ is miniscule and that initially $h^{+} \approx h^{x}$, so as to initiate are write of Equation (4a) above as

$$
\Psi_{4} \cong-\frac{1}{4} \cdot\left[+\partial_{r}^{2} h^{+}\right] \cdot(-1+i) .
$$

The upshot, is that the initial energy flux about the inflationary regime would lead to looking at

$$
\left|\int_{-\infty}^{t} \Psi_{4} \mathrm{~d} t^{\prime}\right| \approx\left|\frac{1}{2} \cdot\left[+\partial_{r}^{2} h^{+}\right]\right| \cdot\left(\tilde{n} \cdot t_{\text {Planck }}\right) .
$$

This will lead to an initial energy flux at the onset of inflation which will be presented as

$$
\frac{\mathrm{d} E}{\mathrm{~d} t}=\left[\frac{r^{2}}{64 \pi}\right] \cdot\left|+\partial_{r}^{2} h^{+}\right|^{2} \cdot\left[\tilde{n} \cdot t_{\text {Planck }}\right]^{2} \cdot \Omega .
$$

If we are talking about an initial energy flux, we then can approximate the above as

$$
E_{\text {initial-flux }} \cong\left[\frac{r^{2}}{64 \pi}\right] \cdot\left|+\partial_{r}^{2} h^{+}\right|^{2} \cdot\left[\tilde{n} \cdot t_{\text {Planck }}\right]^{3} \cdot \Omega_{\text {effective }} .
$$

Inputs into both the expression $\left|\partial_{r}^{2} h^{+}\right|$, as well as $\Omega_{\text {effective }}$ will comprise the rest of this document, plus our conclusions. The derived value of $\Omega_{\text {effective }}$ as well as $E_{\text {initial-flux }}$ 
will be tied into a way to present energy per graviton, as a way of obtaining $n_{f}$. The $n_{f}$ value so obtained, will be used to make a relationship, using Y. J. Ng's entropy [7] [8] counting algorithm of roughly $S_{\text {entropy }} \sim n_{f}$. We assert that in order to obtain $S_{\text {entropy }} \sim n_{f}$ from initial graviton production, as a way to quantify $n_{f}$, that a small mass of the graviton can be assumed.

How to tie in this energy expression, as given in Equation (8) will be to look at the formation of a non trivial gravitional measure which we can state as a new big bang for each of the $\mathrm{N}$ universes as represented by [19] and $n\left(E_{i}\right)$ the density of states at a given energy $E_{i}$ for a partition function defined by

$$
\left\{\Xi_{i}\right\}_{i=1}^{i \equiv N} \propto\left\{\int_{0}^{\infty} \mathrm{d} E_{i} \cdot n\left(E_{i}\right) \cdot \mathrm{e}^{-E_{i}}\right\}_{i=1}^{i=N}
$$

Each of the terms $E_{i}$ would be identified with Equation (8) above, with the following iteration given, namely for $N$ universes

$$
\left.\left.\frac{1}{N} \cdot \sum_{j=1}^{N} \Xi_{j}\right|_{j \text {-before-nucleation-regime }} \stackrel{\text { vacuum-nucleation-tranfer }}{\longrightarrow} \Xi_{i}\right|_{i \text {-fixed-after-nucleation-regime }} \text {. }
$$

For $N$ number of universes, with each $\left.\Xi_{j}\right|_{j \text {-before-nucleation-regime }}$ for $j=1$ to $\mathrm{N}$ being the partition function of each universe just before the blend into the RHS of Equation (10) above for our present universe. Also, each of the independent universes given by $\left.\Xi_{j}\right|_{j \text {-before-nucleation-regime }}$ would be constructed by the absortion of say one million black holes sucking in energy. i.e. in the end.

$$
\left.\left.\Xi_{j}\right|_{j \text {-before-nucleation-regime }} \approx \sum_{k=1}^{M a x} \tilde{\Xi}_{k}\right|_{\text {black-holes-jth-universe }}
$$

One can treat Equation (10) as a de facto Ergotic mixing of prior universes to a present universe, with the partition function of each of the universes defined by Equation (9) above.

Filling in the imputs into Equation (9) to Equation (11) is what will be done in the months aghead. $\left|\partial_{r}^{2} h^{+}\right|$will be the one to fill in, via considering [20] plus other models. Doing so will begin to allow us to form more precise evaluations of Equation (9) to Equation (11).

For the sake of convenience, one can write [21] [22]

$$
\left|\partial_{r}^{2} h^{+}\right| \sim k^{2} h^{+}
$$

So, then

$$
E_{\text {initial-flux }} \sim\left[\frac{r^{2}}{64 \pi}\right] \cdot k^{4} \cdot\left[h^{+}\right]^{2} \cdot\left[\tilde{n} \cdot t_{\text {Planck }}\right]^{3} \cdot \Omega_{\text {effective }}
$$

For our purposes, we shall call $r \sim l_{\text {Planck }} \propto 10^{-34} \mathrm{~cm}, t_{\text {Planck }} \sim 10^{-44} \mathrm{sec}, \Omega_{\text {effective }}$ an effective cross sectional area as to the emission of gravitons, and $k$ defined as a physical wave vector. L. Crowell stated that GW would undergo massive red shifting [23]. Needless to state, the value of $k$ to consider would be for the $\mathrm{GHz}$ band of GW [21] [22]. 


$$
\left(k \approx k_{G W}\right)^{2} \gg\left|\frac{1}{a} \cdot \frac{\mathrm{d}^{2} a}{\mathrm{~d} \eta^{2}}\right|
$$

Also, for the frequencies of [21] [22] $10^{9}-10^{10} \mathrm{~Hz}$, then

$$
h \sim h_{r m s} \sim 10^{-30}-10^{-34} .
$$

Then the numerical count factor can either be of two times, either as a bit count, or just as straight for a primordial black hole.

Namely, if a net acceleration is such that $a_{a c c e l}=2 \pi k_{B} c T / \hbar$ as mentioned by Verlinde [22] [23], as an Unruh result, and that the number of "bits" is

$$
n_{\mathrm{Bit}}=\frac{\Delta S}{\Delta x} \cdot \frac{c^{2}}{\pi \cdot k_{B}^{2} T} \approx \frac{3 \cdot(1.66)^{2} g^{*}}{\left[\Delta x \cong l_{p}\right]} \cdot \frac{c^{2} \cdot T^{2}}{\pi \cdot k_{B}^{2}} .
$$

This Equation (16) has a $T^{2}$ temperature dependence for information bits, as opposed to $[5]$

$$
S \sim 3 \cdot\left[1.66 \cdot \sqrt{\tilde{g}_{*}}\right]^{2} T^{3} \sim n_{f} .
$$

Should the $\Delta x \cong l_{p}$ order of magnitude minimum grid size hold, then conceivably when $T \sim 10^{19} \mathrm{GeV}[24]$

$$
n_{\mathrm{Bit}} \approx \frac{3 \cdot(1.66)^{2} g^{*}}{\left[\Delta x \cong l_{p}\right]} \cdot \frac{c^{2} \cdot T^{2}}{\pi \cdot k_{B}^{2}} \sim 3 \cdot\left[1.66 \cdot \sqrt{\tilde{g}_{*}}\right]^{2} T^{3} .
$$

The situation for which one has [24] [25] $\Delta x \cong l^{1 / 3} l_{\text {Planck }}^{2 / 3}$ with $l \sim l_{\text {Planck }}$ corresponds to $n_{\text {Bit }} \propto T^{3}$ whereas $n_{\text {Bit }} \propto T^{2}$ if $\Delta x \cong l^{1 / 3} l_{\text {Planck }}^{2 / 3} \gg l_{\text {Planck }}$.

Here, we make ths assumption that either $\tilde{n} \sim n_{\text {Bit }} \propto T^{2}$ or $\tilde{n} \sim n_{\text {Bit }} \propto T^{3}$ per unit volume of phase space with the temperature $T$ varying from a low value to up to $10^{34}$ Kelvin (Planck temperature scale). All these scaling parameters would be placed in Equation (13) above, with Equation (13) then put in a discretized version of Equation (9), Equation (10), and Equation (11)

\section{Conclusion, Organizing Inputs into Finding the Mapping for Equation (10), from Black HOLES}

Job one will be in determining if $\tilde{n} \sim n_{\text {Bit }} \propto T^{2}$ or $\tilde{n} \sim n_{\text {Bit }} \propto T^{3}$ per unit volume of phase space with the temperature $T$ varying from a low value to up to $10^{34} \mathrm{Kelvin}$ (Planck temperature scale). Once this would be established, then coming up with details of Equation (10) mapping would be feasible. The author views this as a way to establish if there is an ergotic mixing protocol of millions of black holes from different universes. The details of this mapping, as specified as an investigative protocol, where a discretization of Equation (19) would be necessarily part of the physics research work. Also, it would necessitate making a linkage to what Beckwith et al. put up as far as a numerical count for "massive" graviton counts in a per unit phase space volume of a GW detector which can be written as [20] [26].

$$
J_{\text {effective }} \cong n_{\text {count }} \cdot m_{4 \text {-D-Gravition }}
$$


As stated by Beckwith, in [27], $m_{\text {4-D-Gravition }} \sim 10^{-65}$ grams, while $n_{\text {count }}$ is the number of gravitons which may be in the detector sample. Getting Equation (19) straight for a detector while understanding the inter relationship of $n_{\text {count }}$ to $\tilde{n} \sim n_{\text {Bit }} \propto T^{2}$ or $\tilde{n} \sim n_{\text {Bit }} \propto T^{3}$ per unit volume of phase space initially is what we should be doing.

We should be able to make such an identification; the material/procedures outlined in the following document would be appropriate to review as far as new ways to outline the new methods brought up, above. We hope to do so as soon as circumstances permit [28], as has been worked in Chongqing, China.

Finally, the issues brought up by Appendix E, as to first of all fidelity with respect to LIGO and Gravitational waves, as well as the foundations of gravity brought up by Dr. Corda need to be vetted. Once this is done, and the formulas held to be approximate, it is conceivable that the datum and speculations given by Dr. Corda as referenced in Appendix E will be examinable and hopefully confirmed. In short, we would require an enormous "inflation" style $\phi$ valued scalar function, and an initial almost vanishingly small initial scale factor of the order of say $a^{2}(t) \sim 10^{-110}$, and this requires careful vetting of the issues, brought up in Appendix E below. The issues of Appendix E need to be examined as to confirm the usefulness of the generalization of the Penrose models so brought up, in this document.

\section{Acknowledgements}

This work is supported in part by National Nature Science Foundation of China grant No. 11375279.

\section{References}

[1] Penrose, R. (2007) Conformal Cyclic Cosmology, Dark Matter, and Black Hole Evaporation. IGC Inaugural Conference, Penn State University, State College, 7-11 August 2007.

[2] Beckwith, A.W. (2008) Penrose Model Potential, Compared with Coleman-Weinberg Potential for Early Universe Scalar Evolution. Electronic Journal of Theoretical Physics, 5, 95 106.

[3] Traschen, J. (2000) An Introduction to Black Hole Evaporation. Published in Mathematical Methods of Physics, Proceedings of the 1999 Londrina Winter School, World Scientific. http://arxiv.org/abs/gr-qc/0010055

[4] Hawking, S.W. (1992) Evaporation of Two-Dimensional Black Holes. Physical Review Letters, 69, 406-409. http://dx.doi.org/10.1103/PhysRevLett.69.406

[5] Lloyd, S. (2002) Computational Capacity of the Universe. Physical Review Letters, 88, Article ID: 237901. http://dx.doi.org/10.1103/physrevlett.88.237901

[6] Carroll, S. (2004) An Introduction to General Relativity Space Time and Geometry. Addison Wesley Publishing house, San Francisco.

[7] Ng, Y.J. (2008) Spacetime Foam: From Entropy and Holography to Infinite Statistics and Nonlocality. Entropy, 10, 441-461. http://dx.doi.org/10.3390/e10040441

[8] Ng, Y.J. (2008) Quantum Foam and Dark Energy. International Workshop on the Dark Side of the Universe.

http://ctp.bue.edu.eg/workshops/Talks/Monday/QuntumFoamAndDarkEnergy.pdf

[9] Giovannini, M. (2008) A Primer on the Physics of the Cosmic Microwave Background. 
World Press Scientific, Singapore. http://dx.doi.org/10.1142/6730

[10] Hunt, P. and Sakar, S. (2004) Multiple Inflation and the WMAP "Glitches". Physical Review D, 70, Article ID: 103518. http://dx.doi.org/10.1103/physrevd.70.103518

[11] Ruutu, V., Eltsov, V., Gill, A., Kibble, T., Krusius, M., Makhlin, Y.G., Placais, B., Volvik, G. and Wen, Z. (1996) Vortex Formation in Neutron-Irradiated ${ }^{3} \mathrm{He}$ as an Analog of Cosmological Defect Formation. Nature, 382, 334-336. http://dx.doi.org/10.1038/382334a0

[12] Hingsaw, G. (2007) Private Communications. IUCAA Meeting, Pune.

[13] Beckwith, A.W. (2008) Toward a Viable Dark Matter Candidate without Invoking SUSY. Talk Given in IDM 2008, Sweden. http://agenda.albanova.se/contributionDisplay.py?contribId=369\&amp;sessionId=254\&am p; confId $=355$

[14] Beckwith, A.W. (2008) Several Routes for Determining Entropy Generation in the Early Universe, Links to CMBR Spectra, and Relic Neutrino Production. Proceedings of $43 \mathrm{rd}$ Rencontres de Moriond: Cosmology, La Thuile, 15-22 March 2008, 353-354.

[15] Tcharkian, D.H. (2008) Presentation “Gravitating Yang-Mills Fields" at Bremen, August 29th, 2008, at the "Models of Gravity in Higher Dimensions", August 25-29th, 2008. 418 WE-Heraeus-Seminar (To Appear in Proceedings Which the Seminar Will Put out in Later 2008-2009).

[16] Penrose, R. (2006) Before the Big Bang: An Outrageous New Perspective and Its Implications for Particle Physics. Proceedings of EPAC, Edinburgh, 2759-2763.

[17] Sanchez, N. (2007) 11th Paris Cosmology Colloquium, August 18th, 2007 with Respect to Sanchez, N. Understanding Inflation and Dark Energy in the Standard Model of the Universe, "D. Chalonge” School. http://chalonge.obspm.fr/Programme2007.html

[18] Beckwith, A.W. (2011) Energy Content of Gravitation as a Way to Quantify Both Entropy and Information Generation in the Early Universe. Journal of Modern Physics, 2, 58-61. http://dx.doi.org/10.4236/jmp.2011.22010

[19] Hamber, H.W. (2009) Quantum Gravitation, the Feyman Path Integral Approach. Springer-Verlag, Heidelberg.

[20] Beckwith, A.W., Li, F.Y., Yang, N., Dickau, J., Stephenson, G. and Glinka, L. (2011) Is Octonionic Gravity Relevant near the Planck Scale? http://vixra.org/abs/1101.0017

[21] Li, F., Tang, M. and Shi, D. (2003) Electromagnetic Response of a Gaussian Beam to High Frequency Relic Gravitational Waves in Quintessential Inflationary Models. Physical Review D, 67, 104008. http://dx.doi.org/10.1103/PhysRevD.67.104008

[22] Li, F. and Yang, N. (2009) Phase and Polarization State of High Frequency Gravitational Waves. Chinese Physics Letters, 26, 050402. http://dx.doi.org/10.1088/0256-307X/26/5/050402

[23] Crowell, L. (2011) Private Communication.

[24] Beckwith, A.W. and Glinka, L. (2010) The Arrow of Time Problem: Answering if Time Flow Initially Favouritizes One Direction Blatantly. Prespacetime Journal, 1, 1358-1375. http://vixra.org/abs/1010.0015

[25] Verlinde, E.P. (2011) On the Origin of Gravity and the Laws of Newton. Journal of High Energy Physics, 2011, 29. ArXiv:1001.0785v1[hep-th] http://dx.doi.org/10.1007/JHEP04(2011)029

[26] Beckwith, A.W. (2010) Talk as Given in Chongqing University Department of Physics, 4 November 2010.

[27] Beckwith, A.W. (2010) Applications of Euclidian Snyder Geometry to the Foundations of 
Space-Time Physics. Electronic Journal of Theoretical Physics, 7, 241-266.

[28] Clive Woods, R., Baker Jr., R.M.L., Li, F.Y., Stephenson, G.V., Davis, E.W. and Beckwith, A.W. (2011) A New Theoretical Technique for the Measurement of High-Frequency Relic Gravitational Waves. Submitted for Possible Publication. http://vixra.org/abs/1010.0062 http://dx.doi.org/10.4236/jmp.2011.26060

[29] Sakar, S. (2008) Private Communications to Beckwith.

[30] Peiris, H.V., Komatsu, E., Verde, L., Spergel, D., Bennett, C., Halpern, M., Hinshaw, G., Jarosik, N., Kogut, A., Limon, M., Meyer, S., Page, L., Tucker, G., Wollack, E. and Wright, E. (2003) First-Year Wilkinson Microwave Anisotropy Probe (WMAP) Observations: Implications for Inflation. The Astrophysical Journal Supplement Series, 148, 213-231. http://dx.doi.org/10.1086/377228

[31] Sakar, S. (2016) http://www-thphys.physics.ox.ac.uk/people/SubirSarkar/

[32] Torrieri, G. and Mishustin, I. (2008) Instability of Boost-Invariant Hydrodynamics with a QCD Inspired Bulk Viscosity. Physical Review C, 78, 021901(R).

http://arxiv.org/abs/0805.0442

[33] Asakawa, M., Bass, S.A. and Müller, B. (2006) Anomalous Viscosity of an Expanding Quark-Gluon Plasma. Physical Review Letters, 96, 252301. http://dx.doi.org/10.1103/PhysRevLett.96.252301

[34] Li, F.Y., Yang, N., Fang, Z.Y., Baker, R.M.L., Stephenson, G.V. and Wen, H. (2009) Signal Photon Flux and Background Noise in a Coupling Electromagnetic Detecting System for High-Frequency Gravitational Waves. Physical Review D, 80, 064013. http://dx.doi.org/10.1103/physrevd.80.064013

[35] http://quantumgravity.aei.mpg.de/

[36] Smoot, G. (2007) 11th Paris Cosmology Colloquium, August 18th, 2007 with Respect to Smoot, G. CMB Observations and the Standard Model of the Universe, "D. Chalonge" School. http://chalonge.obspm.fr/Programme2007.html

[37] Beckwith, A.W. (2007) Several Routes for Determining Entropy Generation in the Early Universe, Links to CMBR Spectra, and Relic Neutrino Production. 6th International Conference on Gravitation and Cosmology (ICGC-2007), Ganeshkhind, Pune, 17-21 December 2007, 1-18; Also Presented at KITP, UCSB. http://online.itp.ucsb.edu/online/partcosmo_c08/beckwith/

[38] Kolb, E. and Turner, S. (1994) The Early Universe. Westview Press, Chicago.

[39] Corda, C. (2012) Primordial Gravity's Breath. Electronic Journal of Theoretical Physics, 9 , 1-10.http://arxiv.org/abs/1110.1772

[40] Corda, C. (2008) Massive Gravitational Waves from the $\mathrm{R}^{2}$ Theory of Gravity: Production and Response of Interferometers. International Journal of Modern Physics A, 23, 15211535. http://dx.doi.org/10.1142/S0217751X08038603

[41] Corda, C. (2009) Interferometric Detection of Gravitational Waves: The Definitive Test for General Relativity. International Journal of Modern Physics D, 18, 2275-2282.

http://arxiv.org/abs/0905.2502 http://dx.doi.org/10.1142/S0218271809015904

[42] Beckwith, A. (2016) Gedanken Experiment Examining How Kinetic Energy Would Dominate Potential Energy, in Pre-Planckian Space-Time Physics, and Allow Us to Avoid the BICEP 2 Mistake. Journal of High Energy Physics, Gravitation and Cosmology, 2, 75-82. http://dx.doi.org/10.4236/jhepgc.2016.21008

[43] Gao, C. (2012) A Model of Nonsingular Universe. Entropy, 14, 1296-1305. 
http://dx.doi.org/10.3390/e14071296

[44] Abbott, B.P., et al. (LIGO Scientific Collaboration and Virgo Collaboration) (2016) Observation of Gravitational Waves from a Binary Black Hole Merger. Physical Review Letters, 116, 061102. https://physics.aps.org/featured-article-pdf/10.1103/PhysRevLett.116.061102

[45] Abbott, B.P., et al. (LIGO Scientific Collaboration and Virgo Collaboration) (2016) GW151226: Observation of Gravitational Waves from a 22-Solar-Mass Binary Black Hole Coalescence. Physical Review Letters, 116, 241103.

http://dx.doi.org/10.1103/PhysRevLett.116.241103 


\section{Appendix A: Variations in the CMBR Spectra and What They Imply for Entropy Production}

Our guess is as follows: the matter-energy flux implied by the existence of a wormhole accounts for perhaps $10^{7}$ bits of information. These could be transferred via a wormhole solution from a prior universe to our present, and there could be perhaps $10^{120}$ minus $10^{7}$ bits of information temporarily suppressed during the initial bozonification phase of matter right at the onset of the big bang itself.

Then we predict that there is a dramatic drop in the degrees of freedom during the beginning of the descent of temperature from about $T \approx 10^{32}$ Kelvin to at least three orders of magnitude less. The drop in degrees of freedom happens as we move out in time from an initial red shift, $z \approx 10^{25}$, to something lower, which is when the temperature drops from about $T \approx 10^{32}$ Kelvin to a significantly lower value of [17]

$$
T \approx \sqrt{\varepsilon_{V}} \times 10^{28} \text { Kelvin } \sim T_{\text {Hawkings }} \cong \frac{\hbar \cdot H_{\text {initial }}}{2 \pi \cdot k_{B}}
$$

which model we can come up with that does this is the one we need to follow, experimentally. And it gives us hope of confirming whether or not we can eventually analyze the growth of structure in the initial phases of quantum nucleation of emergent spacetime. We also need to consider the datum so referenced for the irregularities of the cooling-down phase of inflation, as mentioned by Sakar [29] in an e mail to the author, Beckwith,

"Quasi-DeSitter space-time during inflation has no "lumpiness" - it is necessarily very smooth. Nevertheless one can generate structure in the spectrum of quantum fluctuations originating from inflation by disturbing the slow-roll of the inflation-in our model this happens because other fields to which the inflation couples through gravity undergo symmetry breaking phase transitions as the universe cools during inflation."

The race track models, after the inflation begins to decline, would be ideal in obtaining the necessary couplings between the inflation, and fields which undergo a symmetry breaking transformation. We will refer to this topic in a future publication. We can make a few observations though about the assumed coupling. First, there is a question of whether there is a finite or infinite fifth dimension. String theorists have argued for a brane world with a warped, infinite extra dimension, allowing for the inflation to decay into the bulk so that after inflation, the effective dark energy disappears from our brane. This is achieved by shifting away the decay products into the infinity of the 5th dimension. Nice hypothesis, but it presumes $\mathrm{CMB}$ density perturbations could have their origin in the decay of a MSSM flat direction. It would reduce the dynamics of the inflation if there were separation between a $D p$ brane and $\bar{D} p$ antibrane via a moduli argument. that if we do not have an infinite fifth dimension? What if it is compacted only? We then have to change our analysis. Another thing. We place limits on inflationary models; for example, a minimally coupled $\lambda \phi^{4}$ is disfavored at more than $3 \sigma$. Result? Forget quartic inflationary fields, as has been shown by Peiris, Hingshaw et al. [30]. We 
can realistically hope that WMAP will be able to parse through the race track models to distinguish between the different candidates. So far, "First-Year Wilkinson Microwave Anisotropy Probe (WMAP)1 Observations: Implications For Inflation" is giving chaotic inflation a run for its money.

Figure A1 by Sarkar shows the glitches that need to be addressed in order to make a CMBR data set congruent with an extension of the standard model of cosmology. Passed to the author, February 2008 [30], and brought up in IDM 2008 in a way which was communicated to the author by Sakar [31].

\section{Appendix B: Formulation of Criteria for a Second-Order Phase Transition at the Onset of Nucleation of a New Universe}

Let us first review Torrieri's and Mushuntin's [32] contribution to stability analysis of a wave functional treatment of a QCD bulk viscosity-over-entropy constant-ratio state equation. The idea is that we have initially a super hot plasma reaching a peak value of viscosity for a given temperature $\mathrm{T}$, which is less than or equal to a critical temperature, $T_{C}$ reflecting the QCD plasma having a peak value for viscosity. For those who wish to understand how this may work out, we can refer to a paper by Asakawa et al. [33] which specified a sheer bulk viscosity approximated by a viscosity value with $d_{f} \approx O(100)$, which weakly depends upon the number of quark flavors $n_{f}$ in the quark-gluon plasma

$$
\eta_{C}=\left[d_{f} \cdot T^{3} / g^{4} \ln g^{-1}\right]
$$

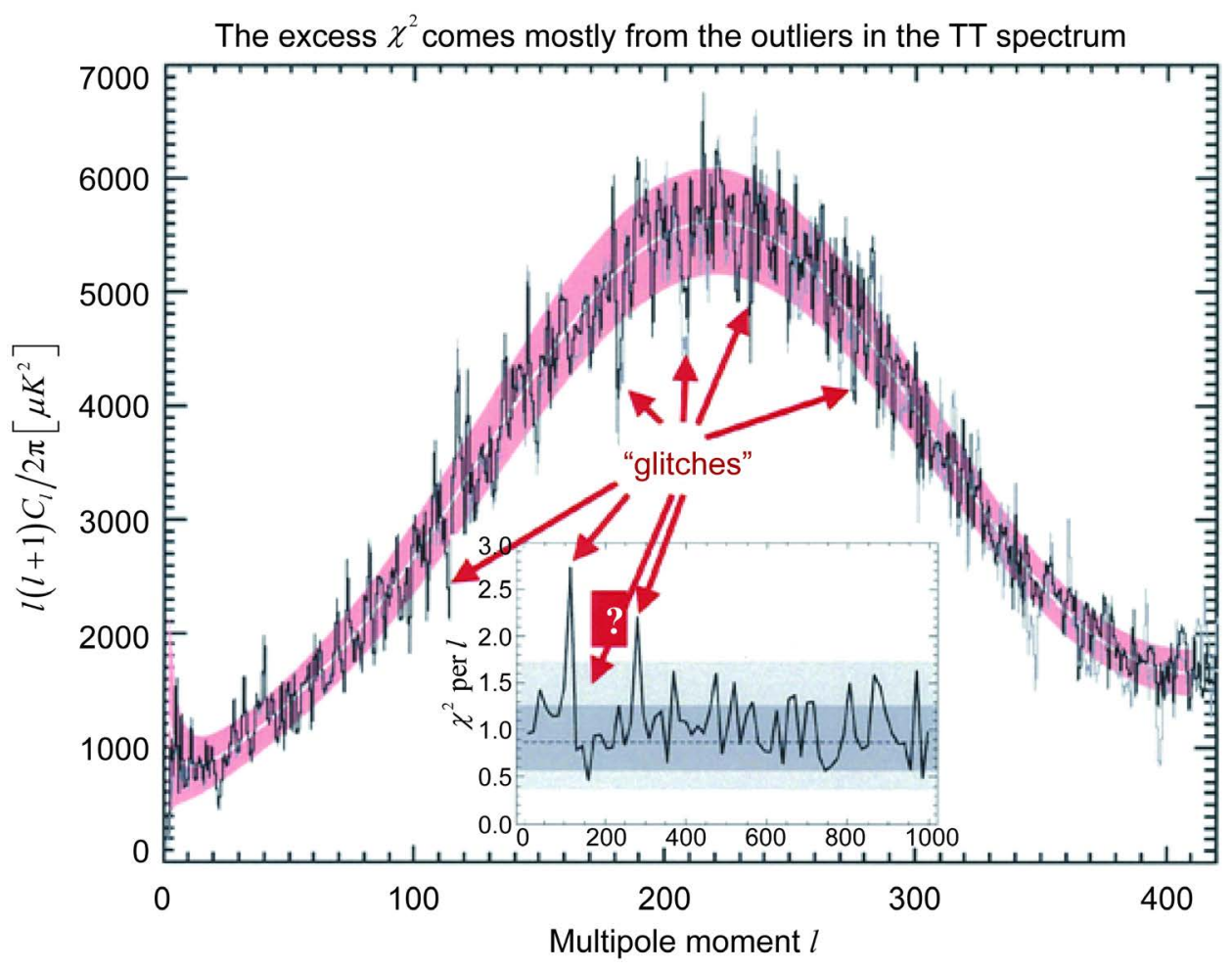

Figure A1. Is the primordial density perturbation really scale-free? 
Here, $g$ is fixed by the number of degrees of freedom of the system. Asakawa et al. [33] also specify that in a quark-gluon plasma, frequently there is an additional anomalous contribution to viscosity, $\eta_{A}$ caused by turbulent fields within the quarkgluon plasma. Asakawa et al. [33] concluded in their document that frequently we have

$$
\eta_{\text {Total }}^{-1}=\eta_{C}^{=1}+\eta_{A}^{-1}
$$

Frequently we also have for extremely high temperatures to a good first approximation,

$$
S_{\text {Density }}=\frac{2 \cdot \pi^{2}}{45} \cdot g_{*} \cdot T^{3}
$$

where $g_{*}$ is the net degrees of freedom of the plasma gas that we can model as an ultra-relativistic fluid. For high temperatures, if $g_{*}$ is on the order of 100 , i.e., reflecting many initial degrees of freedom,

$$
\eta_{\text {Total }} / s_{\text {Density }} \approx \text { const } \sim[1 / 4 \pi] .
$$

With classical fluid models, even for quark-gluon plasmas, this assumes we are working with $\eta_{A}^{-1}$ as not a very strong contributing factor to Equation (B2), leading to almost infinite viscosity if we have viscosity almost entirely dependent upon temperature, as the temperature climbs.

With the model of entropy so offered above, we have if the temperature is not elevated and the two terms in Equation (B2) contribute, trouble in obtaining a stable value for Equation (B4) above as a constant. It so happens that Torrieri's and Mushuntin's [32] idea is to incorporate a modification of the Bjorken equation for cosmology applications,

$$
\tau^{-3} \frac{\mathrm{d}\left[\tau^{3} s\right]}{\mathrm{d} \tau}=\frac{3 s}{R \tau}
$$

where $\tau$ is conformal time, and $R$ is the Reynolds number, and $s$ is entropy density. This Equation (B5) is well above the complexity level of what one expects from the simple linearized models, where we look at, say, if $y$ represents space time "length," etc., with

$$
s(\tau)=s_{0}(\tau)+\delta \cdot s(\tau, y) \exp [i k y]
$$

And a velocity $v \propto x / t$ so that eventually we look at $x_{1}=\delta \cdot s / s$ and $x_{2} \equiv y-y_{\text {space-time }}$. So the stability analysis we have is

$$
\tau \frac{\partial}{\partial \tau}\left[\begin{array}{l}
x_{1} \\
x_{2}
\end{array}\right] \equiv\left[\begin{array}{ll}
A_{11} & A_{12} \\
A_{21} & A_{22}
\end{array}\right]\left[\begin{array}{l}
x_{1} \\
x_{2}
\end{array}\right] .
$$

This is when we have at high temperatures a major simplification of the $A_{i j}$ terms in the matrix in the right hand side of Equation (B7). This simplification of the right hand side of Equation (7) happens when we write $\eta \approx T^{3}$ and $s \propto T^{3}$. We obtain with this simplification of entropy and viscosity a relatively constant Reynolds number $R_{0}$, and a relatively constant speed of "sound" in the viscous media $c_{s}^{0}$. The resulting sim- 
plification and drop out of terms in the evolution equation allows us to write [32] [33]

$$
A_{11}=c_{s}^{02} R_{0}^{-1}
$$

and

$$
A_{12}=-k \cdot\left(1-2 R_{0}^{-1}\right)
$$

and

$$
A_{21}=k c_{s}^{02} \cdot\left(1-3 R_{0}^{-1}\right) /\left(1-R_{0}^{-1}\right)
$$

and

$$
A_{22}=-\left[\left(1-c_{s}^{02}\right)+c_{s}^{02} R_{0}^{-1}+3 c_{s}^{02} R_{0}^{-1}\left(1-R_{0}^{-1}\right)+k^{2} \cdot R_{0}^{-1}\right] /\left(1-R_{0}^{-1}\right) .
$$

In this limit we have a stability analysis performed for the eigenvalues of

$$
A+A^{\mathrm{T}}
$$

where we are using $A \equiv\left[\begin{array}{ll}A_{11} & A_{12} \\ A_{21} & A_{22}\end{array}\right]$, and with the summarized results that for $\left\{\lambda_{\min }, \lambda_{\max }\right\}$ of Equation (B12) are such that, if

$$
\begin{aligned}
& \lambda_{\min }>0 \text { we always have instability } \\
& \lambda_{\max }<0 \text { we always have stability } \\
& \lambda_{\min }<0, \lambda_{\max }>0, \text { we some times have stability, }
\end{aligned}
$$

and sometimes we do not have stability.

The forms of Equation (B13) to Equation (B15) remain the same, but we assert that if we deviate from strict adherence to $\eta \approx T^{3}$ and $s \propto T^{3}$ due to marked initial conditions, i.e., unusual contributions due to the an harmonic contribution to viscosity $\eta_{A}$ we will have increasingly involved criteria for forming the matrix for Equation (B12) and Equation (B7) to Equation (10). We are looking into what these criteria should be for very unstable initial GUT criteria, with the proviso that we are not able to use simple linearization in GUT initial conditions, but that the ratio of $\eta_{\text {Total }} / s_{\text {Density }} \sim[1 / 4 \pi]$ holds [32] [33].

\section{Appendix C: Comparing Implementation of Jack Ng's $\Delta S \approx \Delta N$ for Wavelengths Cubed, of the Order of Magnitude of an Entropy Generating Volume of Space, with Giovannini's Calculation of Entropy for All Permissible Ranges of Frequencies}

As stated above, our implementation of the $\Delta S \approx \Delta N$ rule for HFGW [7] [8] assumes we are able to make a direct comparison between the wavelength of HFGWs and the region of space in which they are evaluated. This comparison yields an interpretation of a growth of entropy due to an infusion of vacuum energy at the onset of inflation, which we think needs to be falsified experimentally. i.e., that in the beginning of quantum nucleation, there were perhaps $10^{7}$ bits of information present. That the production of relic gravitons in a HFGW early universe nucleation environment perhaps added up to $10^{30}$ bits of information in $10^{-10}$ seconds-perhaps closer to an order of mag- 
nitude of $10^{-35}$ seconds in the boost effects of entropy from information transferred from a prior universe to our present universe. The analysis for how this could happen depends upon the verification of a supposition that HFGWs have a wavelength whose value cubed would be within an order of magnitude of the initial volume of space-time in which the HFGW are nucleated in relic inflationary conditions.

Saying this though leads us to consider: do all frequencies contribute to the generation of gravitational waves equally? (This has implications for the generation of entropy, for reasons we will get to next.)

On the face of it, this question is nonsense. LISA and LIGO, two very well engineered detectors, are superb detectors of low frequency gravitational waves, as was given by the Amaldi 5 (2007) meeting [30]. In addition, the betting is that allegedly that signal/noise issues will make detection of HFGWs, especially from relic conditions, exceptionally difficult. The Li-Baker design effort, with its emphasis on a static magnetic field that can be impinged upon by HFGWs has a ready answer to this alleged difficulty. However, the sheer number of contributions to entropy if all ranges of frequencies contribute to GW production in the universe should be considered [34].

Fortunately, there is a calculation authored by Giovannini [9] and others that does count to entropy generation in total from the entire spectrum of GW generated, with a startling conclusion: that the present high level of entropy today can be effectively generated by GW production! This calculation reads as follows. If we set $\mathrm{V}$ as the spacetime volume, then look at $v_{0} \sim 10^{-18} \mathrm{~Hz}$, and $v_{1} \sim 10^{11}\left(H_{1} / M_{P}\right)^{3 / 2} \sim 10^{11} \mathrm{~Hz}$ as an upper bound, assuming no relationship like the $\mathrm{GW}$ wavelength cubed, as proportional to early universe volume, which leads to $r(v) \equiv \ln \bar{n}_{\text {gravitons }}$, where $\bar{n}_{\text {gravitons }}$ refers to the number of produced gravitons over a very wide spectral range of frequencies. This assumes that we are working with $H_{1} \propto M_{P}$

$$
S_{g w}=V \cdot \int_{v_{0}}^{v_{1}} r(v) \cdot v^{2} \mathrm{~d} v \cong\left(10^{29}\right)^{3} \cdot\left(H_{1} / M_{P}\right)^{3 / 2} \approx 10^{87}-10^{88} .
$$

This should be compared with HFGW production in relic conditions

$\left.\Delta S\right|_{\text {relic-HFGW }} \approx \Delta N \sim 10^{21}$ right after the onset of nucleation of a new universe. i.e. there is have relic gravitational production, as occurring after the $2^{\text {nd }}$ order initial phase transition referenced in Appendix B, for a GUT, with information/entropy for universe which Dr. Smoot pegs as less than or equal to $10^{7}$-information/10 5 -entropy $\underset{\text { 2nd-order-phase-transition }}{\longrightarrow} 10^{120}$-information $/ 10^{88}$-entropy in our present universe, which will be explained more fully in future publications.

This should be compared with the result that Sean Carroll [6] came up with: that for the universe as a whole

$$
S_{\text {Total }} \sim 10^{88} .
$$

This Equation (C2) should be compared with the even odder result that the author discussed in a question and answer period in the Bad Honnef perspectives in quantum gravity [35] meeting, April 2008 to reconcile Equation (C2) with the odd prediction 
given in Equation (C3) namely, as presented by Carroll, [6]

$$
S_{\text {Black-Hole }} \sim 10^{90} \cdot\left[\frac{M}{10^{6} \cdot M_{\text {Solar-Mass }}}\right]^{2} \text {. }
$$

i.e. the black hole in the center of our galaxy may have purportedly more entropy than the entropy of the entire KNOWN universe.

Our hierarchy of how to generate entropy from initial conditions present in the initial cosmological evolution is an attempt to make sense of the inherent weirdness present in Equation (C1), Equation (C2), and Equation (C3). The three equations together do not fit as a consistent whole. We assert that there is no way that we can meaningfully justify the conclusions of Equation (C1). And while we view graviton production as crucially important for the rise in entropy, as outlined by Dr. Smoot [36], graviton production is most likely to be concentrated as narrow relic graviton production as an onset to entropy generation.

We hope that the articles following this manuscript will enable us to handle the frankly physically absurd implications inherent in all three of the basic equations written in this document and permit us to develop an experimentally falsifiable set of experimental procedures to reasonably investigate entropy creation from first principles.

\section{Appendix D: Emergent Inflation “Field" Due to Thermal Input from a Prior Universe (The D'Albembertain Operation in an Equation of Motion for Emergent Scalar Fields)}

This was presented at the IUCAA meeting in India by the author, Beckwith, in December 2007 [37] and Beckwith [2].

We begin with the D'Albertain operator as part of an equation of motion for an emergent scalar field. We refer to the Penrose potential (with an initial assumption of Euclidian flat space for computational simplicity) to account for, in a high temperature regime, an emergent non-zero value for the scalar field $\phi$ due to a zero effective mass at high temperatures.

When the mass approaches far lower values is when a non-zero scalar field reappears.

Let us now begin to model the Penrose quintessence scalar field evolution equation. Look at the flat space version of the evolution equation

$$
\ddot{\phi}-\nabla^{2} \phi+\frac{\partial V}{\partial \phi}=0 \text {. }
$$

In the Friedman-Walker metric, this uses the following as a potential system to work with, namely:

$$
V(\phi) \sim-\left[\frac{1}{2} \cdot\left(M(T)+\frac{\Re}{6}\right) \phi^{2}+\frac{\tilde{a}}{4} \phi^{4}\right] \equiv-\left[\frac{1}{2} \cdot\left(M(T)+\frac{\kappa}{6 a^{2}(t)}\right) \phi^{2}+\frac{\tilde{a}}{4} \phi^{4}\right] .
$$

This assumes $\kappa \equiv \pm 1,0$, and a curvature signature compatible with an open universe. 
That means $\kappa=-1,0$ as possibilities. So we will look at the $\kappa=-1,0$ values, beginning with

$$
\begin{aligned}
& \ddot{\phi}-\nabla^{2} \phi+\frac{\partial V}{\partial \phi}=0 \\
& \Rightarrow \phi^{2}=\frac{1}{\tilde{a}} \cdot\left\{c_{1}^{2}-\left[\alpha^{2}+\frac{\kappa}{6 a^{2}(t)}+M(T)\right]\right\} \\
& \Leftrightarrow \phi \equiv \mathrm{e}^{-\alpha \cdot r} \exp \left(c_{1} t\right)
\end{aligned}
$$

We find the following basic phenomena, namely

$$
\begin{aligned}
& \phi^{2}=\frac{1}{\tilde{a}} \cdot\left\{c_{1}^{2}-\left[\alpha^{2}+\frac{\kappa}{6 a^{2}(t)}+\left(M(T) \approx \varepsilon^{+}\right)\right]\right\} \underset{M(T \sim \text { high }) \rightarrow 0}{\stackrel{\longrightarrow}{\longrightarrow}} \phi^{2} \neq 0 \\
& \phi^{2}=\frac{1}{\tilde{a}} \cdot\left\{c_{1}^{2}-\left[\alpha^{2}+\frac{\kappa}{6 a^{2}(t)}+\left(M(T) \neq \varepsilon^{+}\right)\right]\right\} \underset{M(T \sim \text { Low }) \neq 0}{\stackrel{\underset{M}{\longrightarrow}}{\longrightarrow}} \phi^{2} \approx 0 .
\end{aligned}
$$

The difference is due to the behavior of $M(T)$. We use $M(T) \sim$ axion mass $m_{a}(T)$ in asymptotic limits with Kolb's [38]

$$
m_{a}(T) \cong 0.1 \cdot m_{a}(T=0) \cdot\left(\Lambda_{Q C D} / T\right)^{3.7}
$$

\section{Appendix E. Foundations of Gravity, Inflation Physics, and Also a Variant of If or Not There Is a Singular Universe. As Well as Fidelity to the LIGO Results, Shown by Abbot et al.}

We here reference the following, i.e. Dr. Corda investigated if the inflation had a given trace effect in relic inflation astrophysical measurements, in [39]. This may lead to a reshaping of the initial conditions assumed in the beginning of inflationary physics, even within the form of the modified Penrose Cyclic conformal cosmology paradigm. We need to investigate this, and to see if also our modified version of the Penrose cyclic conformal cosmology paradigm is in itself confirmation of, or rejection of the $\mathrm{R}^{\star *} 2$ theory of gravity given in [40]. Furthermore, the question of if there is a scalar-tensor origin to gravity, or if the initial phases of the inflationary expansion, as given by Corda in [41] are strictly in accordance to the usual general relativistic theory will affect how we evaluate datum represented in Figure A1. i.e. this really needs to be looked at. The question of if Kinetic energy is bigger than Potential energy in the Pre Planckian space time, as given in [42] by Beckwith, will affect the emergence of inflation physics, and possibly give support to Corda's [39] treatise as to the nature of the "gravity's breath" treatment of the inflation. The question of if there is a singular or non singular universe, was raised in [43] and our continued model building should either confirm or falsify the Gao hypothesis of a non singular universe. Finally, we also state that our work should be in support of Abbot's LIGO summary of the consequences of GW generation, as to the initial generation of gravity waves as given in [44] and its follow up in [45].

Once the caveats, and necessary investigations of the references, [39]-[45] are done 
we will be on our way toward confirming if we are on the verge of turning gravitational wave astronomy into a valuable research tool which can confirm, or deny the generalization of the Penrose Cyclic conformal cosmology model so elaborated in this manuscript.

Submit or recommend next manuscript to SCIRP and we will provide best service for you:

Accepting pre-submission inquiries through Email, Facebook, LinkedIn, Twitter, etc. A wide selection of journals (inclusive of 9 subjects, more than 200 journals)

Providing 24-hour high-quality service

User-friendly online submission system

Fair and swift peer-review system

Efficient typesetting and proofreading procedure

Display of the result of downloads and visits, as well as the number of cited articles Maximum dissemination of your research work

Submit your manuscript at: http://papersubmission.scirp.org/ 